\title{
Color confinement and the Faddeev-Popov ghosts in Coulomb gauge QCD
}

\author{
Yoshiyuki Nakagawa* \\ Research Center for Nuclear Physics, Osaka University \\ Ibarakisi, Osaka 567-0044, Japan \\ E-mail: nkgwercnp.osaka-u.ac.jp
}

\section{Atsushi Nakamura}

Research Institute for Information Science and Education, Hiroshima University

Higashi-Hiroshima 739-8521, Japan

E-mail: nakamuraeriise.hiroshima-u.ac.jp

\section{Takuya Saito}

Integrated Information Center, Kochi University

Kochi, 780-8520, Japan

E-mail: tsaitoulkochi-u.ac.jp

\section{Hiroshi Toki}

Research Center for Nuclear Physics, Osaka University

Ibarakisi, Osaka 567-0044, Japan

E-mail: toki@rcnp.osaka-u.ac.jp

\begin{abstract}
We investigate the Gribov-Zwanziger scenario in the Coulomb gauge using a SU(3) quenched lattice gauge simulation. The dressing function of the ghost propagator diverges in the infrared limit. This result is expected from the fact that the Faddeev-Popov eigenvalue density gets concentrated near the vanishing eigenvalue compared to that in the abelian gauge theory. The turnover of the transversal gluon propagator is not observed up to the largest lattice volume explored in this study. The instantaneous part of the time-time component of the gluon propagator which corresponds to the color-Coulomb potential in the continuum limit diverges stronger than the simple pole. Furthermore, we observe that the ghost propagator show good scaling while both components of the gluon propagator do not show scaling in the considered range of the lattice spacing.
\end{abstract}

The XXV International Symposium on Lattice Field Theory

July 30 - August 42007

Regensburg, Germany

\footnotetext{
*Speaker.
} 


\section{Introduction}

To understand the mechanism of color confinement is a challenging issue in particle and nuclear physics. There have been several scenarios proposed for color confinement. In the GribovZwanziger and the Kugo-Ojima confinement scenarios [1, 2], the Faddeev-Popov (F-P) ghosts play a significant role in the mechanism of color confinement.

About ten years ago, the Landau gauge study of the gluon and the ghost Dyson-Schwinger equations (DSEs) revealed that the ghost propagator diverges in the infrared (IR) limit stronger than the simple pole, and the gluon propagator is suppressed in the IR region and vanishes at zero momentum [3]. Furthermore, it was demonstrated that the contribution of the ghost loop in the gluon DSE is crucial for the IR suppression of the gluon propagator. Although the ghost contribution is numerically small in the perturbation theory, the IR dynamics of the Yang-Mills theory is dominated by the unphysical ghost degrees of freedom, i.e., 'IR ghost dominance'.

In the Coulomb gauge, the importance of the F-P ghosts and the confinement scenario are more transparent. The Coulomb gauge is a physical gauge and the Hamiltonian can be decomposed into two parts,

$$
\begin{aligned}
H= & \frac{1}{2} \int d^{3} x\left\{\left(E_{i}^{\mathrm{tr}}\right)^{2}+B_{i}^{2}\right\} \\
& +\frac{1}{2} \int d^{3} y \int d^{3} z \rho^{a}(\vec{y}, t) \mathscr{V}^{a b}\left(\vec{y}, \vec{z} ; A^{\mathrm{tr}}\right) \rho^{b}(\vec{z}, t) .
\end{aligned}
$$

The first term represents the energy of the transverse components of the color-electric and colormagnetic fields. The second part, the instantaneous interaction energy between color charges, originates from the energy of the longitudinal color-electric fields. $\rho$ is the color charge density

$$
\rho^{a}=g f^{a b c} A_{i}^{b, \text { tr }} E_{i}^{c, \text { tr }}+\rho_{\text {quark }}^{a}
$$

and $\mathscr{V}$ the kernel of the instantaneous interaction

$$
\mathscr{V}^{a b}\left(\vec{y}, \vec{z} ; A^{\mathrm{tr}}\right)=\left(M^{-1}[A]\left(-\nabla^{2}\right) M^{-1}[A]\right)_{\vec{y}, \vec{z}}^{a b}
$$

whose vacuum expectation value is called the color-Coulomb potential, and $M$ the F-P ghost operator

$$
M^{a b}=-\partial_{i} D_{i}^{a b}=-\delta^{a b} \partial_{i}^{2}-g f^{a b c} A_{i}^{c, \text { tr }} \partial_{i} .
$$

From the partition function with the Coulomb gauge Hamiltonian, one can evaluate the time-time component of the gluon propagator composed by the instantaneous part and the non-instantaneous part [4],

$$
g^{2}\left\langle A_{4}(x) A_{4}(y)\right\rangle=g^{2} D_{44}(x-y)=V(\vec{x}-\vec{y}) \delta\left(x_{4}-y_{4}\right)+P(x-y) .
$$

It was shown by Zwanziger that the instantaneous color-Coulomb potential provides an upper bound for the static potential and the necessary condition for the static potential being a confining potential is that the color-Coulomb potential is also a confining potential [5]. Recently lattice QCD simulation showed that the instantaneous color-Coulomb potential rises linearly at large distances and it is stronger than the static potential; this is an expected result from the Zwanziger's inequality 
$[6,7]$. Therefore, in the Coulomb gauge, the color confinement is attributed to the instantaneous color-Coulomb interaction.

In the Gribov-Zwanziger confinement scenario, the strong confining feature of the instantaneous interaction originates from the strong long range correlation of F-P ghosts. As Gribov discussed, the Coulomb gauge does not fix a gauge completely, and the gauge configurations are restricted to the Gribov region where the F-P operator is positive [8]. On the boundary of the Gribov region, so-called the Gribov horizon, the lowest eigenvalue of the F-P operator vanishes. It was argued by Zwanziger that entropy favors gauge configurations near the Gribov horizon and the eigenvalue distribution of the F-P operator gets concentrated near the vanishing eigenvalue compared to that in the abelian gauge theory [9]. Such an enhancement has been observed by the recent lattice simulations $[10,11]$. Accordingly, the ghost propagator becomes more singular than the free propagator and the IR enhancement of the ghost propagator leads to the long-range interaction of the color-Coulomb potential which is responsible for the color confinement.

The analysis of the ghost DSE in the Coulomb gauge has revealed that the equal-time transverse gluon propagator vanishes at zero momentum if the ghost dressing function diverges stronger than $1 / \sqrt{|\vec{p}|}[12]$. The vanishing of the equal-time transverse gluon propagator would imply that gluons have infinite effective mass in the Coulomb gauge, which indicates the confinement of gluons. By contrast, the time-time component of the gluon propagator would diverges stronger than the simple pole in the IR limit since it contains the instantaneous color-Coulomb potential, which is responsible for the color confinement.

In this study we perform a lattice investigation of the IR behavior of the ghost and the gluon propagators in order to clarify the confinement mechanism in the Coulomb gauge. A similar work done by Voigt et. al has also been reported in this conference [13].

\section{Lattice observables}

On a lattice, the F-P operator is an $8 V_{3} \times 8 V_{3}$ sparse matrix ( $V_{3}$ is the lattice 3-volume) and expressed in terms of $\mathrm{SU}(3)$ spatial link variables $U_{i}$ as

$$
\begin{aligned}
M_{x y}^{a b}=\sum_{i} \mathfrak{R e} \operatorname{Tr}[ & \left\{T^{a}, T^{b}\right\}\left(U_{i}(x)+U_{i}(x-\hat{i})\right) \delta_{x, y} \\
& \left.-2 T^{b} T^{a} U_{i}(x) \delta_{y, x+\hat{i}}-2 T^{a} T^{b} U_{i}(x-\hat{i}) \delta_{y, x-\hat{i}}\right] .
\end{aligned}
$$

The ghost propagator is defined as the vacuum expectation value of the inverse of the F-P ghost operator,

$$
G^{a b}(\vec{x}-\vec{y})=\left\langle\left(M^{-1}[A]\right)_{\vec{x}, \vec{y}}^{a b}\right\rangle
$$

In contrast with the abelian gauge theory, the Green's function $M^{-1}$ of the F-P ghost operator depends on the gauge fields $A_{i}^{a}$. Since the ghost propagator is diagonal in color space, the ghost propagator in the momentum space can be written as

$$
G^{a b}(\vec{p})=\delta^{a b} \frac{Z_{G}(|\vec{p}|)}{\vec{p}^{2}} .
$$

Here $Z_{G}$ is the dressing function of the ghost propagator. The non-trivial momentum dependence of the ghost propagator due to the quantum correction is encoded in the dressing function. 
The equal-time gluon propagator is given by

$$
D_{\mu \nu}^{a b}(\vec{x}-\vec{y})=\left\langle A_{\mu}^{a}(\vec{x}) A_{\nu}^{b}(\vec{y})\right\rangle=D_{\mu \nu}^{a b}(\vec{x}-\vec{y}),
$$

and the transversal equal-time gluon propagator in the momentum space can be written as

$$
D_{i j}^{a b}(\vec{p})=\delta^{a b}\left(\delta_{i j}-\frac{p_{i} p_{j}}{\vec{p}^{2}}\right) D^{\operatorname{tr}}(|\vec{p}|)
$$

The instantaneous part of the time-time component of the gluon propagator is

$$
D_{44}^{a b}(\vec{p})=\delta^{a b} \frac{Z_{44}(|\vec{p}|)}{\vec{p}^{2}},
$$

which corresponds to the color-Coulomb potential $V$ in the continuum limit.

The lattice propagators depends on the magnitude of the three momentum $|\vec{p}|$ and the lattice cutoff $\Lambda$. In this study, the lattice propagators are renormalized multiplicatively at $\mu=3[\mathrm{GeV}]$.

\section{Results and discussions}

We calculate the ghost propagator and the gluon propagator by the $S U(3)$ lattice gauge simulations in a quenched approximation. The lattice configurations are generated by the heat-bath Monte Carlo technique with the Wilson plaquette action. In these simulations we adopt the iterative method to fix a gauge. In order to obtain the ghost propagator we have inverted the F-P matrix by the conjugate gradient method and used plane wave sources. The details of the calculation will be published elsewhere.

\subsection{Ghost propagator}

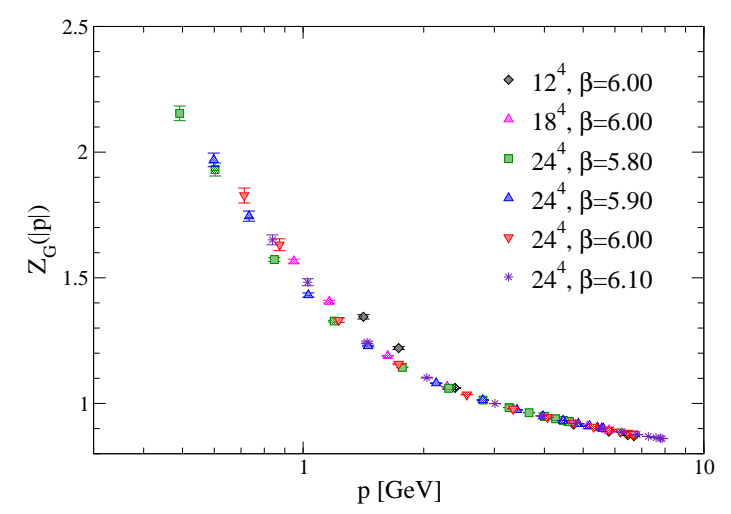

Figure 1: The ghost dressing function in the confinement phase. As a renormalization condition, we set $Z_{G}(|\vec{p}|=3[\mathrm{GeV}])=1$.

In Fig. 1 the ghost dressing function is plotted as a function of the magnitude of the three momentum. Although the finite volume effect is seen at small lattice volume, the propagator shows good scaling. As is expected in the Gribov-Zwanziger scenario, the ghost dressing function shows 
a divergent behavior in the IR limit, which results in the confining feature of the color-Coulomb instantaneous interaction.

In order to explore the momentum dependence of the ghost dressing function in the IR region, we fitted data below $1[\mathrm{GeV}]$ on $24^{4}$ lattice at $\beta=5.80$ to the IR power law ansatz

$$
Z_{G}(|\vec{p}|)=\frac{c_{1}}{\left(\vec{p}^{2}\right)^{\gamma_{g h}}}
$$

We found the IR exponent

$$
\gamma_{g h}=0.29(2), \quad c_{1}=1.42(1)
$$

with $\chi^{2} / n d f=0.244$. The analysis of the ghost DSE in the Coulomb gauge has revealed that the equal-time transversal gluon propagator vanishes at zero momentum if the ghost IR exponent exceeds 0.25 [12]. From our result of the ghost dressing function we expect that the equal-time transversal gluon propagator vanishes at $\vec{p}=0$, which indicates the confinement of gluons.

\subsection{Equal-time transverse gluon propagator}
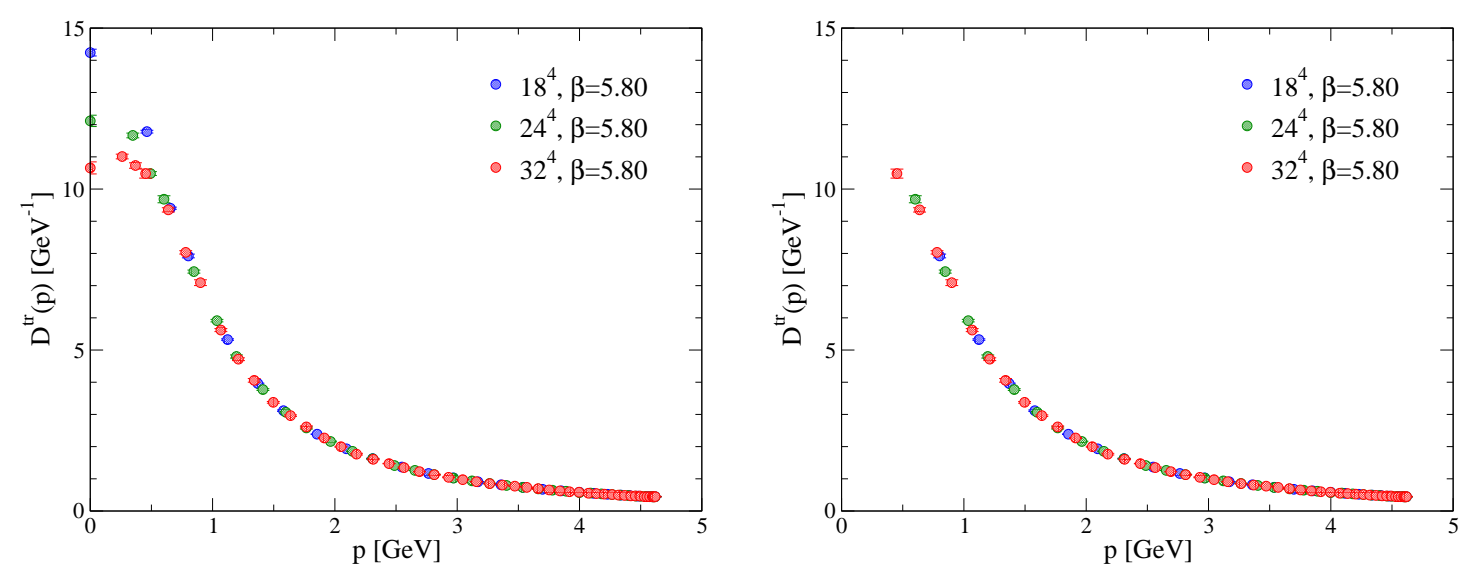

Figure 2: The equal-time transverse gluon propagator without (left) and with (right) the cone cut.

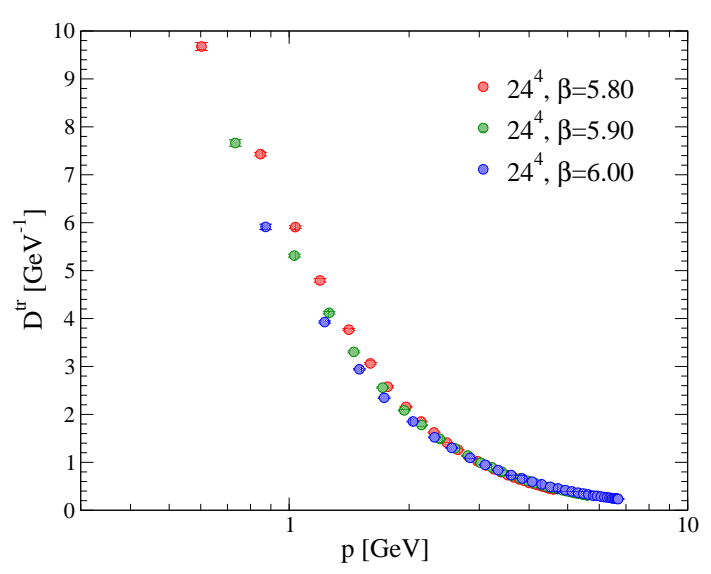

Figure 3: The equal-time transversal gluon propagator at various lattice couplings. 
The lattice simulation results for the transversal gluon propagator are presented in Fig. 2. We find that the finite volume effect is removed by applying the cone cut [14]. The turnover of the transversal gluon propagator is not observed up to the largest lattice volume explored in this study. Fig. 3 shows the transversal gluon propagator at different lattice couplings. We observe that the transversal propagator does not show scaling and is faced with the strong finite lattice spacing effect. This has also been observed by Voigt et al. [13].

\subsection{Instantaneous part of the time-time gluon propagator}

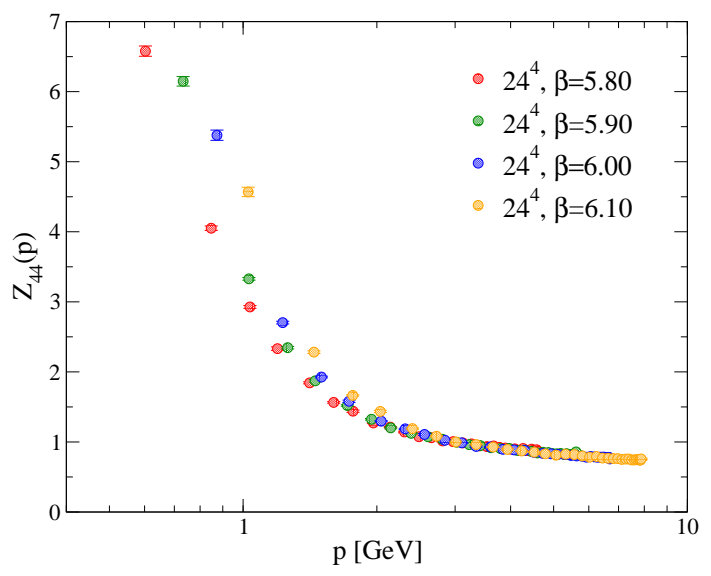

Figure 4: The instantaneous part of the time-time gluon propagator at various lattice couplings. Both the cylinder and the cone cut are applied.

The dressing function of the instantaneous part of the time-time gluon propagator is illustrated in Fig. 4. We see that the dressing function diverges in the IR region, and this is consistent with the fact that the color-Coulomb potential obtained by measuring the partial Polyakov line correlator shows a confining behavior. Scaling is not seen for the instantaneous time-time gluon propagator as well as the transversal gluon propagator. Non-scaling of the instantaneous time-time gluon propagator was also observed in the $\mathrm{SU}(2)$ lattice gauge simulations [15].

\section{Summary and conclusion}

We investigated the ghost and the gluon propagators in the Coulomb gauge using the quenched lattice simulations. We found that the ghost dressing function diverges in the infrared limit. Such an IR divergent behavior of the F-P ghosts leads to the strong long-range instantaneous interaction which confines color charges. The ghost IR exponent was found to be about 0.29 ; this implies the vanishing equal-time gluon propagator at vanishing momentum. The equal-time transversal gluon propagator does not show turnover up to the largest lattice volume explored in this study. Both the transversal and the time-time component of the gluon propagators do not show scaling in the considered range of the lattice coupling constant, and the renormalization procedure must be reconsidered. 


\section{Acknowledgements}

The simulation was performed on SX-5 and SX-8(NEC) vector-parallel computers at the RCNP of Osaka University. We appreciate the warm hospitality and support of the RCNP administrators. One of the authors (Y.N.) acknowledges the support from the Osaka University Scholarship for Short-term Student Dispatch Program.

\section{References}

[1] D. Zwanziger, Nucl. Phys. B399, 477 (1993).

[2] T. Kugo and I. Ojima, Prog. Theor. Phys. Suppl. 66, 1 (1979).

[3] L. von Smekal, R. Alkofer and A. Hauck, Phys. Rev. Lett. 79, 3591 (1997), [hep-ph/9705242].

[4] D. Zwanziger, Prog. Theor. Phys. Suppl. 131, 233 (1998), [hep-th/9802180].

[5] D. Zwanziger, Phys. Rev. Lett. 90, 102001 (2003), [hep-lat/0209105].

[6] J. Greensite and S. Olejnik, Phys. Rev. D67, 094503 (2003), [hep-lat/0302018].

[7] A. Nakamura and T. Saito, Prog. Theor. Phys. 115, 189 (2006), [hep-lat/0512042].

[8] V. N. Gribov, Nucl. Phys. B139, 1 (1978).

[9] D. Zwanziger, Nucl. Phys. B412, 657 (1994).

[10] J. Greensite, S. Olejnik and D. Zwanziger, JHEP 05, 070 (2005), [hep-lat/0407032].

[11] Y. Nakagawa, A. Nakamura, T. Saito and H. Toki, Phys. Rev. D75, 014508 (2007), [hep-lat/0702002].

[12] C. S. Fischer and D. Zwanziger, Phys. Rev. D72, 054005 (2005), [hep-ph/0504244].

[13] A. Voigt, E.-M. Ilgenfritz, M. Mueller-Preussker and A. Sternbeck, PoS LAT2007, 338 (2007), [arXiv:0709.4585 [hep-lat]].

[14] D. B. Leinweber, J. I. Skullerud, A. G. Williams and C. Parrinello, Phys. Rev. D 58, 031501 (1998).

[15] A. Cucchieri and D. Zwanziger, Phys. Rev. D 65, 014001 (2001). 